\title{
Tipo de liderazgo de jefes de oficina y rendimiento laboral de trabajadores administrativos
}

\section{Type of office chief leadership and job performance of administrative workers}

\author{
Raúl Rabanal Oyarce ${ }^{1}$, Carmen Rosa Huamán Muñoz ${ }^{2}$
}

\section{RESUMEN}

La investigación tuvo como objetivo determinar la relación entre liderazgo de jefes de oficina del área administrativa y el rendimiento laboral de los trabajadores de la Universidad Nacional Toribio Rodríguez de Amazonas - 2018, la población estaba constituido por el 100\% de los trabajadores administrativos, la muestra 12 Jefes y 34 trabajadores, para la recolección de datos se utilizó el cuestionario de liderazgo multifactorial (Multifactor Leadership Questionnaire MLQ) desarrollado por Bass y Avolio, para la variable de desempeño laboral se utilizó el formato de evaluación de desempeño laboral del personal del hospital San José - Callao. Los resultados indicaron que existe relación directa entre liderazgo $(60.86 \%)$ y rendimiento laboral $(\mathbf{7 3 . 1 3 \%})$, con influencia significativa, donde predomina el liderazgo transformacional de los jefes de oficina que participaron en la muestra al exhibir conductas tanto de un líder transformacional como de uno transaccional, tomando en consideración las características de la situación, del contexto organizacional y exhibiendo en menor frecuencia comportamientos de liderazgo laissez faire. Se encontró evidencias de trabajadores con un estilo de liderazgo que ayudan a la consecución de las metas y objetivos de la institución, sin la intervención del jefe.

Palabras clave: Liderazgo, liderazgo transformacional, liderazgo transaccional, liderazgo laissez faire, rendimiento laboral.

\begin{abstract}
La investigación tuvo como objetivo determinar la relación entre los jefes de dirección de la oficina del área administrativa y el servicio laboral de los trabajadores de la Universidad Nacional Toribio Rodríguez de Amazonas - 2018, la población estaba constituido por el 100\% de los trabajadores administrativos, la muestra 12 Jefes y 34 trabajadores, para la recolección de datos se utilizó el liderazgo medio gráfico multifactorial (Cuestionario de Liderazgo Multifactor MLQ desarrollado) variable de desempeño laboral se utilizael el formato de evaluación de la laboral del personal del hospital San José - Callao. Los resultados indicaron que existe relación entre liderazgo $(60,86 \%)$ y el liderazgo laboral $(73,13 \%)$, con influencia significativa, donde predomina el liderazgo transformacional de los jefes de oficina que participaron en la muestra al exhibir conductas tanto de un líder transformacional como de uno transaccional, tomando en consideración de las características de la situación, del contexto organizacional y exhibiendo en menor frecuencia confrecuencias de liderazgo laissez faire. Se en evidenciaevidencias de trabajadores con un estilo de liderazgo que ayudan a la consecución de las metas y objetivos de la institución, sin la intervención del jefe.
\end{abstract}

Keywords: Cocoa, positioning, strategic management, Amazonas, Peru.

${ }^{1}$ Dr. en Gestión Pública y gobernabilidad - Docente en la Facultad de Ingeniería Zootecnista, Agronegocios y Biotecnología- Universidad Nacional Toribio Rodríguez de Mendoza de Amazonas. Correo electrónico: raul.rabanal@untrm.edu.pe

${ }^{2}$ Dra. En Gestión Pública y gobernabilidad - Especialista Administrativo de la Universidad Nacional Toribio Rodríguez de Mendoza de Amazonas. Correo electrónico: Carmen.huaman@untrm.edu.pe 


\section{INTRODUCCIÓN}

Los vertiginosos cambios del mundo actual requieren de nuevas formas de liderazgo que faciliten no solo la conducción de ideas y de acciones sino de la credibilidad de intenciones y confianza en la misión que los líderes representan.

Estos cambios acelerados en el mundo, principalmente por los avances tecnológicos y sociales, llevan asociado un mayor nivel de competencia a lo interno de las organizaciones, lo cual ha generado la inquietud por enfrentar los retos que imponen los cambios a los individuos, las organizaciones y el estado. Por ello se viene presentando en forma evolucionada diversas teorías de liderazgo que conjuntamente con otros factores podrían dar una respuesta a los cambios suscitados (Gutiérrez, 1992).

Se han venido investigando elementos y factores que determinan un mayor o menor grado de productividad de los trabajadores teniendo como antecedentes las investigaciones de otros autores relacionados con el estudio entre los que destacan: la teoría de McGregor (1960) quien observó que el comportamiento del líder frente a sus subordinados depende de lo que el líder piense de ellos. Identifico dos posiciones extremas que denominó Teoría " $\mathrm{X}$ " $\mathrm{y}$ Teoría "Y".

De la misma forma; Da Silva y Rodríguez (2010), Relación entre liderazgo y desempeño de tareas, realizo una investigación Correlacional, no experimental, transversal ha utilizado el instrumento construido por Williams y Anderson (1991). En una población de 545 y una muestra de 161 entre gerentes y representantes de ventas respectivamente, teniendo como conclusiones Bass y Avolio, desarrollan el modelo de liderazgo rango completo, señalando que los líderes pueden exhibir conductas tanto de un líder transformacional como de uno transaccional.

Altuve et al. (1999), Relación entre estilos de liderazgo según el modelo de Bolman y Deal y el desempeño laboral, esta investigación utilizó el instrumento basado en el cuestionario de orientación de liderazgo construido por Lee Bolman (1990) traducido y adaptado a la realizada gerencial Venezolana, por el Centro de Desarrollo Humano y Organizacional, del Instituto de Estudios Superiores de Administración (IESA) en 1993, llegando a las siguientes conclusiones: La distribución de los estilos de liderazgo en la población evidenció que la perspectiva sobre saliente fue la estructural, predominado el manejo tradicional de la gerencia ante situaciones organizacionales, en cierto modo insuficiente para satisfacer las necesidades actuales.
La relación entre las variables fue una correlación positiva débil, debido a las cargas subjetivas, implicadas en las mediciones de los datos.

Brown, et al, (1996) Los factores de Liderazgo Transformacional se relacionan positiva y significativamente con consecuencias organizacionales, tales como: satisfacción laboral, esfuerzo extra, relaciones satisfactorias entre subordinado y líder, efectividad en la unidad de equipo, en el trabajo y en la organización.

Gonzales, A. (2010), "Estilos de liderazgo docente en una institución educativa pública del distrito de Magdalena" y Medina, P. (2010) denominada "El liderazgo Transformacional en los docentes de un colegio cooperativo de la ciudad de Lima" las cuales dirigen el tema a los docentes y solo consideran al director como una figura administrativa.

Se identificaron razones que justifican el estudio de la relación de liderazgo con el rendimiento laboral; Dado que los jefes de oficina del área administrativa de la Universidad en su mayoría son Docentes, los mismos que imparten conocimientos a los alumnos y futuros profesionales líderes que formarán parte en algún momento de las direcciones y jefaturas del sector público y privado de la Región y el país.

La relevancia teórica para este trabajo, radica en que sentará la base para revisiones y contribuciones teóricas a los modelos de liderazgo transformacional, transaccional y laissez faire, ya que la teoría se valida en la medida que sus planteamientos se someten

Constantemente a crítica o examen, sobre todo cuando cambia el contexto cultural en el cual se aplica.

La relevancia metodológica se deriva del hecho de que se pretende colaborar con antecedentes científicos respecto de las propiedades psicométricas de funcionamiento de la técnica y la herramienta aplicada en el contexto de Amazonas.

Por último, la relevancia práctica de este estudio, posibilitará la realización de diagnósticos preliminares, estudios exploratorios y descriptivos, al desconocerse aspectos importantes respecto de cómo se comportan los factores del liderazgo transformacional, transaccional y laissez faire en Amazonas y la generación de proyectos más atingentes a nuestra realidad social.

En actualidad se vive en un mundo competitivo caracterizado por un Contexto social, económico, político, cultural y educacional, donde todo impresiona, y exige la adquisición de modelos, estrategias, enfoques o perspectivas de acción, lo cual enfoca a un Sistema de Administración Publica a una 
práctica administrativa para la acción gerencial. Desde ese enfoque, Lussier y Achua (2005), puntualiza que el liderazgo, "que se ha transferido del pasado, simplemente no es capaz de manejar problemas de este tiempo".

En tal sentido, buscamos Identificar las características de los directivos de la institución pública y el efecto que este genera en sus subordinados. Del cual el problema planteado fue: ¿Cuál es la relación entre liderazgo de jefes de oficina del área administrativa y el rendimiento laboral de los trabajadores de la Universidad Nacional Toribio Rodríguez de Mendoza de Amazonas?

Cuyo objetivo general fue "Determinar la relación entre el liderazgo de jefes de oficina del área administrativa y el rendimiento laboral de los trabajadores"; En la investigación se identifica tres dimensiones de liderazgo: liderazgo transformacional, liderazgo transaccional, liderazgo laissez faire

La Hipótesis planteada "Existe una relación de influencia significativa entre el liderazgo de jefes de oficina del área administrativa y el rendimiento laboral de los trabajadores de la Universidad Nacional Toribio Rodríguez de Mendoza de Amazonas 2018“; Donde se analizan las relaciones entre el liderazgo de los jefes y el rendimiento laboral de los trabajadores desde las opiniones vertidas por los trabajadores y el mismo líder.

\section{MATERIAL Y MÉTODO}

El universo de la población estuvo conformado por el $100 \%$ de los trabajadores de área administrativa. En el cual participaron 12 jefes de área y 34 trabajadores los cuales fueron seleccionados en forma aleatoria y de acuerdo al organigrama de la universidad.

Para la recolección de datos de la variable liderazgo, se utilizó el cuestionario multifactorial (multifactor Leadership Questionnaire MLQ, versión abreviada $5 \mathrm{X})$, De este instrumento se aplicaron las dos versiones, la que está orientada para que responda el propio líder ("Uno mismo" o "auto apreciativa") y la que corresponde a los seguidores ("visto por otro o apreciativa").- el cuestionario consta de 82 ítems que responden bajo una escala tipo Likert. Los cinco puntos de la escala tienen una base numérica de 0-12-3-4, cuya correspondencia cualitativa es: $0=a$ veces, $1=$ Rara vez, $2=$ A veces, $3=$ Con frecuencia, 4= Siempre.

Para la recolección de datos de la variable Rendimiento laboral se aplicó la encuesta de evaluación de desempeño laboral que utilizan los directivos del hospital San José del Callao el mismo que se adecua a nuestro contexto. Este instrumento describe al trabajador en qué tiene desarrollada las competencias como: La orientación a resultados, calidad, relaciones interpersonales, iniciativa, trabajo en equipo y organización. La encuesta consta de 20 ítems, con una escala que va de: Muy bajo a Muy Alto.

Para el procesamiento de los datos de esta investigación, se utilizaron las herramientas de Microsoft Excel 2010 y SPSS Versión 18.

\section{RESULTADOS}

Con la finalidad de probar la hipótesis de estudio, la prueba t de Student permitió, establecer si existían o no diferencias significativas, entres las medias de cada una de las variables con los distintos contrastes realizados

Figura 1. Estadistica de diferencia de medias por dimensiones

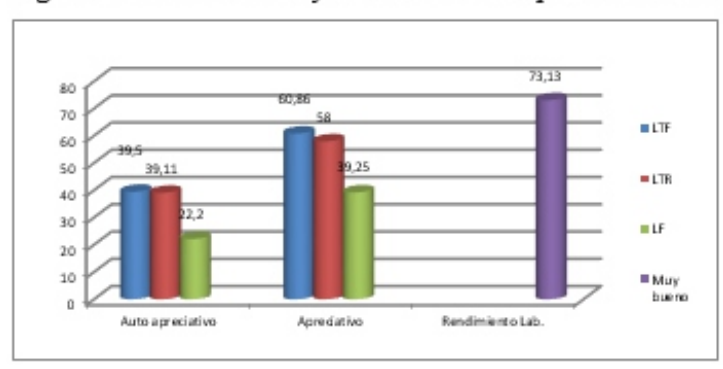

En la figura 1. Diferencia de medias por dimensiones según el puntaje obtenido de los ítems, se observa que la dimensión de liderazgo transformacional "auto apreciativo" tiene una media de 39.59, seguido del liderazgo transaccional con una media de 39.11, lo que indica que los jefes de oficina se auto califican o asumen tener los dos tipos de liderazgo en similar magnitud, diferenciado del liderazgo laisses faire con una media de 22.20 .

Respecto a la dimensión de liderazgo Transformacional "apreciativo" Evaluado por el trabajador tiene una media de 60.86, seguido de liderazgo transaccional con una media de 58 , lo que indica que los trabajadores consideran a sus jefes con un liderazgo transformador en más alto nivel y en menor nivel el liderazgo laisses faire con una media de 39.25 .

De la variable rendimiento laboral de los trabajadores evaluado por los jefes de oficina, encontramos que los trabajadores tienen en promedio un nivel de rendimiento alto con una media de 73.13 en un rango de 30 a 100 puntos, lo que indica que los trabajadores muestran un rendimiento laboral muy bueno según la percepción de sus jefes.

Todas las diferencias de medias entre los estilos de 
Tabla 1. Descriptivos y Prueba $t$ de Student para Liderazgo

\begin{tabular}{|c|c|c|c|c|c|c|}
\hline & \multicolumn{6}{|c|}{ Valor de prueba $=0$} \\
\hline & \multirow[b]{2}{*}{$\mathrm{T}$} & \multirow[b]{2}{*}{ G1 } & \multirow[b]{2}{*}{$\begin{array}{l}\text { Sig. } \\
\text { (bilater } \\
\text { al) }\end{array}$} & \multirow[b]{2}{*}{$\begin{array}{l}\text { Diferenci } \\
\text { a de } \\
\text { medias }\end{array}$} & \multicolumn{2}{|c|}{$\begin{array}{lr}95 \% & \text { Intervalo } \\
\text { de } & \text { confianza } \\
\text { para } & \\
\text { diferencia } & \end{array}$} \\
\hline & & & & & \begin{tabular}{|l} 
Inferio \\
$\mathrm{r}$
\end{tabular} & $\begin{array}{l}\text { Superio } \\
\mathrm{r}\end{array}$ \\
\hline $\begin{array}{l}\text { Liderazgo } \\
\text { visto por } \\
\text { uno mismo }\end{array}$ & 33,442 & 81 &, 000 & 35,243 & 33,147 & 37,3408 \\
\hline $\begin{array}{l}\text { liderazgo } \\
\text { visto por } \\
\text { otros }\end{array}$ & 36,940 & 81 &, 000 & 54,963 & 52,002 & 57,9239 \\
\hline $\begin{array}{l}\text { Rendimient } \\
\text { o laboral de } \\
\text { los } \\
\text { trabajadores }\end{array}$ & 28,561 & 33 &, 000 & 74,411 & 69,111 & 79,7125 \\
\hline
\end{tabular}

liderazgo, resultan estadísticamente significativos, según se aprecia en la Tabla $\mathrm{N}^{\circ} 1$.

Estos datos permiten confirmar la hipótesis alternativa, ya que predomina la tendencia en los jefes de oficina a exhibir comportamientos referidos al liderazgo transformacional y un mayor rendimiento de sus trabajadores.

Tabla 2. Correlaciones de muestras relacionadas

\begin{tabular}{|c|c|c|c|c|}
\hline & & $\mathrm{N}$ & Correlación & Sig. \\
\hline Par 1 & $\begin{array}{l}\text { LTF auto apreciativo y } \\
\text { Rendimiento laboral }\end{array}$ & 22 &,- 054 & ,813 \\
\hline Par 2 & $\begin{array}{l}\text { LTR auto apreciativo y } \\
\text { Rendimiento laboral }\end{array}$ & 18 &,- 072 &, 776 \\
\hline Par 3 & $\begin{array}{l}\text { LF auto apreciativo y } \\
\text { Rendimiento laboral }\end{array}$ & 20 &, 165 & ,486 \\
\hline Par 4 & $\begin{array}{l}\text { LTF apreciativa y } \\
\text { Rendimiento laboral }\end{array}$ & 22 &,- 077 &, 734 \\
\hline Par 5 & $\begin{array}{l}\text { LTR apreciativa y } \\
\text { Rendimiento laboral }\end{array}$ & 18 & ,016 & 949, \\
\hline Par 6 & $\begin{array}{l}\text { LF apreciativa y } \\
\text { Rendimiento laboral }\end{array}$ & 20 & ,548 &, 012 \\
\hline
\end{tabular}

\section{DISCUSIÓN}

Se encontró que los jefes de oficina exhiben comportamientos de estilos de liderazgo transformacional, transaccional y laisses faire, estos comportamientos no se presentan de manera separada, son una combinación entre las diferentes dimensiones de liderazgo prevaleciendo el liderazgo transformacional, seguido del liderazgo transaccional y en menor frecuencia el liderazgo laisses faire, pero cada perfil de Jefe involucra más de uno y menos de otro (Bass y Avolio 2006), es decir va a depender de la capacidad que tenga el jefe de poder interpretar correctamente las características de la situación y del contexto organizacional para mostrar conductas propias de líder.
La dimensión del liderazgo transformacional, da niveles mayores de rendimiento laboral de los trabajadores. Estos datos no revelan que los propios trabajadores tienen su propio liderazgo, en las que direccionan su accionar prospectivamente, a la consecución de metas y objetivos de la institución, con el afán de plasmar sus conocimientos y desarrollarse profesionalmente. El liderazgo ha venido evolucionando por diferentes estilos los cuales unos son la base de otros, caso es el liderazgo transformacional que tiene como base al estilo transaccional, los mismos que estimulan en emerger de la conciencia de los trabajadores, los cuales aceptan y se comprometen con el logro de la misión de la institución, dejando de lado sus intereses personales, para enfocarse a un interés colectivo, a lo que Bass, argumentó que un líder podrá exhibir de acuerdo a las circunstancias y que estas demanden patrones de liderazgo diferente.

El liderazgo transaccional tiene mejor funcionalidad en las empresas privadas, que tienen producciones en masas, lo que es imposible en las instituciones del estado, debido a que ellos tienen sus funciones específicas en cada área. Finalmente consideramos, que esta investigación será un aporte que permitirá contribuir a futuras investigaciones y nuevos métodos de abordaje para los nuevos estilos de liderazgo.

\section{CONCLUSIONES}

Los resultados muestran que las variables contempladas y analizadas en los instrumentos, una relación directa, a mejores actitudes y conductas del líder con el trabajador mejor rendimiento laboral de los mismos.

En la presente investigación se encontró una influencia significativa, donde predomina el liderazgo transformacional de los jefes de oficina, que participaron en la muestra, exhibiendo asimismo y con mayor frecuencia comportamientos correspondientes a diferencia del liderazgo transaccional; se encontró evidencias de trabajadores con un estilo de liderazgo que ayudan a la consecución de las metas y objetivos de la institución, sin la intervención del jefe.

De la hipótesis planteada concluimos que si existe diferencia significativa, con un nivel de significación de $0,5 \%$. Asimismo, se observó niveles altos de desempeño laboral de los trabajadores que participaron en la muestra.

\section{REFERENCIAS BIBLIOGRÁFICAS}

Avolio, B. (1997). The great leadership migration to Full Range Leadership development system. 
Kellogg. Leadership Studies Project: Leadership Transformational, Working Papers. Academy of Leadership Press.

Bass, B. M. y Avolio, B. J. (1990). The implications of transactional and transformational leadership for individual, team, and organizational development. Research in Organizational Change and Development, 4:231-272. Mind Garden, Palo Alto, CA.EUUU. 1995.

Brown W., Birnstihl E. y Wheeler D.: (1996). Leading without authority: an examination of the impact of transformational leadership cooperative extension work groups and teams. Journal of Extension; vol. 34, $\mathrm{N}^{0} 5$.

Da Silva y Rodríguez (2010), Relación entre liderazgo y desempeño de tareas, Tesis de Grado Universidad Católica Andrés Bello, Caracas-Venezuela.

Gonzales, Á (2010) “Estilos de liderazgo docente en una institución educativa pública del distrito de Magdalena". Tesis para optar el grado de Magíster en Educación con mención en Gestión de la Educación. Lima: Pontificia Universidad Católica del Perú, Escuela de Graduados

Gutiérrez O. (1999) La nueva tendencia en liderazgo. Del liderazgo transaccional al transformacional. Revista Marina 1-6. Extraído el 1 de diciembre del 2008.

Lussier, R y Achua C. (2005) Liderazgo: teoría, aplicación, desarrollo de habilidades. Editorial: Cengage Learning

McGregor (1960) El lado humano de las organizaciones: Bogotá: Mac Graw Hill, Edición revisada.

Medina, P. (2010) “El liderazgo transformacional en los docentes de un colegio cooperativo de la ciudad de Lima”. Tesis para optar el grado de Magíster en Educación con mención en Gestión de la Educación. Lima: Pontificia Universidad Católica del Perú, Escuela de Posgrado. 